\title{
UMA PESQUISA SOBRE INTEROPERABILIDADE EM AMBIENTE E-LEARNING COM GAMIFICAÇÃO
}

\author{
Antônio Rege Lopes dos Santos ${ }^{1}$ \\ Alexandre de Sá Carneiro Wanderley²
}

RESUMO: Este artigo apresenta uma pesquisa sobre Interoperabilidade em Ambientes eLearning com Gamificação. Os ambientes e-Learning gamificado tem permitido novos modos de difundir o conhecimento[20]. A gamificação refere-se, ao uso dos elementos de jogos em contexto que "não são de jogos”, e isso, tem chamado a atenção de pesquisadores em investigar sobre o tema. A partir de uma revisão sistemática, este trabalho mostra como resultado, análise de artigos publicados em 2013 e 2017, disponíveis nas bases de dados da IEEE, ACM Library e Google Scholar. Na visão dos autores pesquisados, cresce a motivação e engajamento dos alunos quando utilizam ambientes com gamificação, porém, concordam que, em face da diversidade de sistemas, há necessidade de estudos mais aprofundados sobre como integrar essas plataformas a outros ambientes externos.

Palavra-chave: interoperabilidade. E-Learning. Gamificação.

\section{INTRODUÇÃO}

A educação do século XXI tem como uma das suas principais características a rápida disseminação do conhecimento[I]. A vinda de ambientes e-Learning na academia e nos demais setores da sociedade, já é uma realidade, e tem chamado a atenção de pesquisadores em investigar sobre como esses sistemas podem comunicar a outros sistemas usando técnicas de gamificação[2].

A gamificação em ambientes e-Learning surge como um recurso estratégico voltado para uma ação pedagógica diferenciada, vindo a proporcionar novos modos de difusão do

\footnotetext{
${ }^{1}$ Centro de Estudos Avançados de Recife (CESAR)Recife, Brasil E-mail: regejs@gmail.com.

${ }^{2}$ Centro de Estudos Avançados de Recife(CESAR) Recife, Brasil. E-mail: adscwi6@gmail.com
} 
conhecimento e melhorias no processo de ensino-aprendizagem entre crianças, jovens e adultos[3].

Assim, ao se utilizar dessa [4] mecânica de jogos aplicado a ambientes educacionais, podem gerar interação e motivação do usuário para a realização de metas e tarefas de forma divertida.

Nesse contexto, esse artigo, busca apresentar uma pesquisa sobre a interoperabilidade em ambiente de sistemas e- Learning usando técnicas de gamificação e quais os impactos, essas aplicações têm aumentado o interesse dos alunos e professores para o processo de ensino aprendizagem.

Assim, este trabalho está organizado da seguinte forma. Na seção 2 apresentamos alguns conceitos sobre interoperabilidade, ambientes e-Learning e gamificação. Na seção 3 apresentamos os trabalhos correlatos. $\mathrm{Na}$ seção 4 uma discussão das literaturas pesquisadas. $\mathrm{Na}$ seção 5 o artigo é concluído, bem como a possibilidade de trabalhos futuros.

\section{INTEROPERABILIDADE E-LEARNING E GAMIFICAÇÃO}

A. Interoperabilidade em e-Learning

Atualmente, diversos desenvolvedores procuram[5] criar seus próprios módulos de sistema e-Learning, normalmente esses módulos não conseguem se comunicar com outras plataformas. A necessidade de padronização para conseguir uma melhor interoperabilidade entre os sistemas de e-Learning vem sendo amplamente discutida na academia [6]. Por outro lado, afirma [7] que "quando esses padrões são incorporados nos produtos dos fornecedores, os usuários de e-Learning podem comprar componentes de conteúdo e sistema de vários fornecedores com base na sua qualidade e relevância, com confiança de que eles trabalharão juntos de forma eficaz".

Para os Berenguer e Molina [8], "diversos trabalhos têm tratado de experiências na integração de ferramentas externas em LMS através de LTI, por exemplo, a integração do Google Docs jogos ou laboratórios remotos”, essa integração baseada em LTI e projetada para permitir a vinculação de conteúdo baseado em vídeo em qualquer plataforma de e-Learning, tornando assim Interoperável com outros sistemas [9]. Assim, [10] a interoperabilidade tenta 
resolver o problema usando vários meios para permitir o compartilhamento de conteúdo de aprendizagem. Isso é feito tipicamente criando interfaces que vários LMSs podem usar. Portanto, a interoperabilidade em e-Learning pode ser definido como, diferentes ambientes de aprendizagem, que tenham a capacidade de se comunicar, trocar dados e utilizar a informação que foi trocada[II].

\section{B. Gamificação}

Gamificação é um assunto atual que têm despertado o interesse de vários pesquisadores. Para os autores[12], "gamificação refere-se ao uso dos elementos de jogos em contexto que não são de jogos, para envolver os participantes a fim de melhorar o seu desempenho e passar os desafios apresentados para promover o desenvolvimento das habilidades desejadas". Ampliando esse conceito[13], define que "Gamificação é o uso de estratégias, modelos, dinâmica, mecânica e elementos de jogo em contextos não-jogo, a fim de transmitir uma mensagem ou um conteúdo ou mudar o comportamento através de uma experiência lúdica que promove a motivação, envolvimento e diversão ”. Em outras palavras[I4] os autores reiteram que, "a gamificação é a estratégia que usa a mecânica e as técnicas do jogo para conduzir o comportamento do usuário aumentando a auto contribuição".

Fica claro que gamificação, não está somente em construir modelos ou sistemas, mas também de envolver indivíduos emocionalmente, proporcionando um ambiente que lhe permita a motivação e ao mesmo tempo o engajamento. [15].

Diante disso, podemos definir gamificação como forma criativa de combinação de várias técnicas envolvendo alguns aspectos de estímulos e que se utiliza da mecânica de jogos, com o intuito melhorar diversos ambientes, procurando sempre a motivação para o engajamento de um publico alvo.

\section{TRABALHOS CORRELATOS}

Nesta seção estão relacionados os trabalhos que apontam alguma similaridade a integração de ferramentas ou serviços no tocante a Interoperabilidade em Ambiente e-Learning e Gamificação. 
Os autores[16] propõe um padrão de infraestrutura que pode ser facilmente incorporada a uma API de serviços web REST, o LUDOS "latim ludos, que significa jogos e diversão", utiliza uma abordagem de SOA para realizar a interoperabilidade de dados, serviços e aplicações educacionais, com a premissa de facilitar adoção de gamificação em ecossistemas existentes, além de permitir que diferentes ecossistemas compartilhem aplicações, serviços e recursos. Para o autor, a ferramenta tem um potencial, pois permite auxiliar na criação de vários conteúdos em formatos digitais gamificado, dando a possibilidade de servir como auxilio no processo de ensino e aprendizagem.

Em sua visão Karavirta[6], relata que devido a diversidade de sistemas e-Learning no mercado, a interoperabilidade entre eles, se tornou um problema para usuários que o utilizam esses ambientes, isso acontece, pois, muitos desenvolvedores criam seus próprios módulos do sistema que nem sempre é possível a integração entre eles. Por isso, propõe o um estudo, introduzindo um sistema denominado $\mathrm{A}+$, que tem por finalidade combinar sistemas de avaliação de exercícios, usando uma API de serviços para que outros sistemas possam utilizar. O sistema suporta conceitos tais como cursos, instâncias de curso, exercícios e exercícios com submissões e feedback dentre outros. O A+ usa uma abordagem orientada a serviço de avaliação existentes vagamente acoplado, ou seja, são descrições de atividades e instruções que são definidas em um serviço externo. Para validar a arquitetura proposta, foi utilizado cerca de 140 alunos de um curso de desenvolvimento de software web da Universidade Aalto, que resolveram algumas atividades usando várias linguagens de programação. Os resultados mostraram satisfatórios, pois tecnicamente, o sistema funcionou bem e atendeu o modelo proposto.

Esse artigo[17] apresenta uma solução para sistemas e-Learning baseado na Web, Learning Analytics (LA) e Gamificação chamado de ICT-FLAG. Sendo assim, usa uma ferramenta de Avaliação Formativa (FAT), que proporciona feedback imediato por meio de avaliação e Learning Analytics (LA). Cita também, que a gamificação é usada com o propósito de motivar a participação, bem como aumentar o envolvimento nas atividades. O autor faz uma ressalva, sobre a falta de padrão de interoperabilidade entre as plataformas e-Learning, considera que é possível fornecer um modelo comum de dados, o que permitiria a integração 
entre os conteúdos. Por consequência, dessa escassez, cada sistema tende a usar seu próprio modelo. Por fim, valida seu modelo usando dois testadores e um técnico da Universidade Aberta da Catalunha, que fizeram a integração de uma ferramenta FAT (chamada VerilUOC) com o ICT-FLAG. Os resultados obtidos foram através de quatro indicadores de uma escala o-5, onde 3 obtiveram nota acima da média, o que mostra satisfação em relação ao uso da ferramenta.

No trabalho, os pesquisadores [5], apresentam um sistema para integrar e compartilhar conhecimentos em base de dados de plataformas e-Learning. Propõe, o uso de um middleware que suporta vários motores de busca personalizados. Esses motores saberão onde procurar uma resposta, como filtrá-la e como comparar com as respostas de outras fontes. Assim, busca melhorar e otimizar a relação entre professores e alunos que utilizam essas ferramentas. Porém, apontam que um dos grandes desafios desses serviços e torná-los de interoperáveis, pois na maioria dos casos as plataformas e-Learning não permitem o compartilhamento para outros ambientes. Defendem, que o problema de interoperabilidade não está somente em plataformas e-Learning, mas em outras áreas de conhecimento. Dessa forma, os pesquisadores sem mostraram otimistas, pois o sistema é capaz de se comunicar com várias fontes de conhecimento independentemente da tecnologia.

\section{DISCUSSÃO}

Como se destacou nos trabalhos pesquisados da seção III, percebe-se que alguns autores [5]-[17] citam, a escassez de pesquisas que tratem especificamente questões técnica sobre o tema de Interoperabilidade em Ambientes e-Learning com Gamificação. Assim, foram realizadas algumas pesquisas nas bases de dados da IEEE, ACM Digital Library e Google Scholar para validar o trabalho. A principio, aplicou-se uma busca prévia com palavras-chaves como: Interoperabilidade, ambientes e-Learning e Gamificação. Em seguida, realizamos a segunda etapa com as combinações das palavras utilizando os operadores booleanos (AND) com as seguintes palavras chaves: "Interoperabilidade AND ambientes eLearning AND Gamificação", “Interoperabilidade AND ambientes e-Learning”, 
"Interoperabilidade AND Gamificação", "ambientes e-Learning AND Gamificação”. A Tabela I apresenta os resultados das buscas para cada combinação das palavras.

Tabela I. Ocorrências das buscas por palavras

\begin{tabular}{|l|l|l|l|}
\hline Nome da busca & IEEE & ACM Library & $\begin{array}{l}\text { Google } \\
\text { Scholar }\end{array}$ \\
\hline $\begin{array}{l}\text { Interoperabilidade AND e-Learning } \\
\text { AND Gamificação }\end{array}$ & & & \\
\hline Interoperabilidade AND e-Learning & & & \\
\hline Interoperabilidade AND Gamificação & & & \\
\hline e-Learning AND Gamificação & & & \\
\hline Total (Exceto duplicados) & & & \\
\hline
\end{tabular}

$\mathrm{Na}$ intenção de selecionar os resultados, foram aplicados critérios de inclusão e exclusão, como a de limitação do período de pesquisa entre 2013 e 2017. Os resultados das bases IEEE, ACM Library e Google Scholar se deu pela importância do resultado com a pergunta de pesquisa por meio da leitura dos títulos e em seguida os resumos. Esta etapa resultou em 20 artigos.

Logo após com uma leitura mais especifica dos artigos, excluímos os que não tratavam de similaridade com o tema da pesquisa. Vale ressaltar, que grande parte dos trabalhos foram descartados, pois tratavam de publicações exclusivamente de fatos isolados sobre interoperabilidade, e-Learning e gamificação. Por fim, chagamos ao resultado da seleção com 4 artigos no total que tem uma grande similaridade com esse trabalho, dos quais 3 foram da base de dados IEEE e i do Google Scholar. 
Tabela 2. Filtro totais ocorrências e total de publicações selecionadas

\begin{tabular}{|l|l|l|l|}
\hline Base da busca & Totais de & \multicolumn{2}{|l|}{ Artigos selecionados } \\
\hline ocorrências & & & \\
\hline ACM Library & & & \\
\hline Google Scholar & & & \\
\hline Total & & & \\
\hline Total selecionados & & 4 \\
\hline
\end{tabular}

Percebe-se, na tabela 2 que as pesquisas quando filtradas, ainda não apresentam grande número quando o assunto é Interoperabilidade em Ambientes e-Learning com gamicação, porém, quando se busca pelas palavras interoperabilidade em e-Learning e e-Learning com Gamificação o número de publicações apresentam um número significativo, quando se estende a busca para Interoperabilidade e Gamificação o termo se difere do conceito adotado de se utilizar elementos dentro do contexto da pesquisa. Sendo assim, em todas as ocorrências notou-se a presença dos termos pesquisados, porém, somente I artigo apresentou especificamente[I6] a proposta deste artigo.

Além dos trabalhos pesquisados nas bases de dados IEEE, ACM Library e Google Scholar, é relatado duas aplicações que são utilizadas em ambientes e-Learning com gamificação e usam conexão com outros sistemas. Na primeira temos o Duolingo[18] que é um aplicativo para aprendizagem de línguas de forma divertida de treinar idiomas. Você perde corações se responder errado, avança ao completar unidades, e marca seu progresso a cada conquista. 
Na segunda Trivia Crack [19], uma aplicação voltada para estudos diversas áreas que permite aos usuários competir em questões que são divididas em seis categorias. Essas seis categorias são entretenimento, arte, história, ciência, geografia e esportes. Os usuários podem compartilhar seus resultados, avaliar e traduzir perguntas, desafiar outras pessoas e conversar com elas durante os todo o tempo de acesso.

\section{CONCLUSÃO E TRABALHOS FUTURO}

A proposta deste trabalho teve como base realizar uma revisão sistemática no cenário, onde a Gamificação está presente cada vez mais em ambientes de ensino-aprendizagem eLearning, percebe-se, que um dos grandes desafios dessa modalidade e fazer a integração desses ambientes de forma que haja interoperabilidade entre eles.

As publicações analisadas destacam-se as seguintes vantagens, onde a adoção de ambientes e-Learning com Gamificação é usado como forma de estimular as qualidades cognitivas, sociais, culturais e motivacionais dos participantes, gerando assim, um interesse maior nas atividades propostas, bem como um avanço no processo de aprendizagem.

Os trabalhos futuros é propor uma Arquitetura Interoperável, aplicado a um cenário de gamificação em Ambiente e-Learning, com o propósito de alfabetizar crianças que tenham distúrbios de fala

\section{REFERÊNCIAS}

[I] R. F. da Silva and E. S. Correa, "Novas Tecnologias E Educação: a Evolução Do Processo De Ensino E Aprendizagem Na Sociedade Contemporânea," pp. 23-35, 2014.

[2] D. Kermek, D. Strmecki, M. Novak, and M. Kaniski, "Preparation of a hybrid elearning course for gamification," 2016 39th Int. Conv. Inf. Commun. Technol. Electron. Microelectron. MIPRO 2016 - Proc., pp. 829-834, 2016.

[3] K. Erenli, "The impact of gamification: A recommendation of scenarios for education," 2012 I5th Int. Conf. Interact. Collab. Learn. ICL 2012, 2012.

[4] A. C. de Oliveira, "Gamificação na Educação," Obra Digit., vol. 9, pp. 120-125, 2015.

[5] S. Martín et al., "How to share existing existing knowledge between e-learning 
platforms: Enhancing interoperability," Proc. - 8th IEEE Int. Conf. Adv. Learn. Technol. ICALT 2008, pp. 764-769, 2008.

[6] V. Karavirta, P. Ihantola, and T. Koskinen, "Service-oriented approach to improve interoperability of e-learning systems," Proc. - 2013 IEEE 13th Int. Conf. Adv. Learn. Technol. ICALT 2013, pp. 341-345, 2013.

[7] A. Bakhouyi, "Multiple Criteria Comparative Evaluation on the Interoperability of LMS by Applying COPRAS Method," IEEE Rev. Iberoam. Tecnol. del Aprendiz., no. December, pp. 36r-366, 2016.

[8] D. Perez-Berenguer and J. Garcia-Molina, "An approach for developing interoperable online content,” 2016 Int. Symp. Comput. Educ., pp. I-4, 2016.

[9] M. C. Santos, "SCORM-MPEG : an ontology of interoperable metadata for Multimedia and e-Learning," Comput. Educ. (SIIE), 2016 Int. Symp. 13-I5 Sept. 2016, 2016.

[ı] T. Sirkiä and L. Haaranen, "Acos Server: Towards Smart Learning Content Interoperability," Proc. 15th Koli Call. Conf. Comput. Educ. Res., pp. 169-17o, 2015.

[II] G. S. Santos and J. Jorge, "Interoperable intelligent tutoring systems as open educational resources," IEEE Trans. Learn. Technol., vol. 6, no. 3, pp. 27I-282, 2013.

[12] B. B. Lambruschini and W. G. Pizarro, "Tech - Gamification in university engineering education: Captivating students, generating knowledge," roth Int. Conf. Comput. Sci. Educ. ICCSE 2015, no. Iccse, pp. 295-299, 2015.

[13] F. Largo, F. Duran, C. Arnedo, P. Rosique, R. Cuerda, and R. Carmona, "Gamification of the learning process: lessons learned," IEEE Rev. Iberoam. Tecnol. del Aprendiz., vol. 8540, no. c, pp. I-I, 2016.

[14] A. P. Markopoulos, M. F. Dossis, A. Fragkou, and P. D. Kasidiaris, "Gamifying elearning as a means of overcoming its deficiencies," ACM Int. Conf. Proceeding Ser., vol. 25-27-Sept, pp. 86-91, 2016.

[I5] M. R. Regalado, E. Aranha, and T. R. da Silva, "Gamifying an online approach for promoting game development Learning and contest: An experience report," 2016 IEEE Front. Educ. Conf., pp. I-8, 2016.

[16] W. Silva, F. Campos, R. Braga, and J. David, "LUDOS: uma Infraestrutura para Gamificação em Ecossistemas de E-learning," Brazilian Symp. Comput. Educ. (Simpósio Bras. Informática na Educ. - SBIE), vol. 26, no. I, p. 469, 2015.

[17] D. Gañán, S. Caballé, R. Clarisó, and J. Conesa, "Towards the Effective Software 
Development of an eLearning Platform Featuring Learning Analytics and Gamification Towards the Effective Software Development of an eLearning Platform Featuring Learning Analytics and Gamification,” 2016 3oth Int. Conf. Adv. Inf. Netw. Appl. Work., no. March, pp. 177-182, 2016.

[18] Doualingo "Aprenda idiomas" . 2017 [Online]. Available: https://pt.duolingo.com/. [Acessed: 27-Fev-2017].

[19] Trivia Crack " Learn how to play ". 2017 [Online]. Available: https://www.triviacrack.com/. [Acesssed: 28-02-2017].

[20] M. Sanmugam, Z. Abdullah, H. Mohamed, N. M. Zaid, B. Aris, and H. van der Meijden, "The Impacts of infusing Game elements and Gamification in learning," 8th IEEE Int. Conf. Eng. Educ. (ICEED 2016), pp. 131-136, 2016 